ROCKY MOUNTAIN

JOURNAL OF MATHEMATICS

Volume 31, Number 1, Spring 2001

\title{
GEOMETRICAL PROPERTIES OF THE PRODUCT OF A $C^{*}$-ALGEBRA
}

\author{
KAIDI EL AMIN, ANTONIO MORALES CAMPOY AND \\ ANGEL RODRIGUEZ PALACIOS
}

0. Introduction. The study of the geometry of norm-unital complex Banach algebras at their units [5], [6] takes its first impetus from the celebrated Bohnenblust-Karlin theorem [3] asserting that the unit of such an algebra $A$ is a vertex of the closed unit ball of $A$. As observed in [5, pp. 33-34], the Bohnenblust-Karlin paper contains a stronger result, namely that, for such an algebra $A$, the inequality $n(A, \mathbf{1}) \geq(1 / e)$ holds. Here $\mathbf{1}$ denotes the unit of $A$, and $n(A, \mathbf{1})$ is a suitably defined nonnegative real number which depends only on the Banach space of $A$ and the norm-one distinguished element $\mathbf{1}$. As the main result, we prove in this paper that the product of every nonzero $C^{*}$-algebra $A$ is a vertex of the closed unit ball of the Banach space $\Pi(A)$ of all continuous bilinear mappings from $A \times A$ into $A$. As in the above mentioned case, the vertex property follows from stronger "numerical" conditions. Indeed, if $A$ is a nonzero $C^{*}$-algebra, and if $p_{A}$ denotes the product of $A$, then $n\left(\Pi(A), p_{A}\right)$ is equal to 1 or $1 / 2$ depending on whether or not $A$ is commutative (Theorem 1.1). We note that our main result improves the recent one in [24, Corollary 2.7] asserting that the product of every nonzero $C^{*}$-algebra $A$ is an extreme point of the closed unit ball of $\Pi(A)$.

In Section 2 we show that the main result remains true for the socalled alternative $C^{*}$-algebras (Theorem 2.5). Alternative $C^{*}$-algebras are defined by means of the Gelfand-Naimark abstract system of axioms but relaxing the familiar requirement of associativity to that of alternativity. Alternative $C^{*}$-algebras arise in a natural way in functional analysis. Indeed, Gelfand-Naimark axioms on a general nonassociative unital algebra imply the alternativity $[\mathbf{2 2}$, Theorem 14] (see also [9]) and the existence of alternative $C^{*}$-algebras failing to be associative is well known (see [17, Example 13] and [8, Theorem 3.7]). Alternative $C^{*}$-algebras are studied in detail in $[\mathbf{2 0}]$ and $[\mathbf{8}]$ and have shown

Received by the editors on July 17, 1999.

Copyright (C)2001 Rocky Mountain Mathematics Consortium 
useful in the structure theory of $J B^{*}$-triples [14]. Most tools applied in the extension of the main result to the alternative setting are actually proved for the so-called noncommutative $J B^{*}$-algebras [20]. We note that the class of noncommutative $J B^{*}$-algebras contains that of alternative $C^{*}$-algebras. Moreover, as in the case of these last algebras, noncommutative $J B^{*}$-algebras have a natural birth. Namely, if a norm-unital complete normed nonassociative complex algebra $A$ is subjected to the geometric Vidav condition characterizing $C^{*}$-algebras in the associative context [4, Theorem 38.14], then $A$ is a noncommutative $J B^{*}$-algebra $[\mathbf{2 3}]$.

Finally, in Section 3, we raise the question if alternative $C^{*}$-algebras are the unique noncommutative $J B^{*}$-algebras $A$ whose products are vertices of the closed unit ball of $\Pi(A)$. In relation to this question, we exhibit an example showing that, if the vertex property is relaxed to the extreme point property, then the answer is negative (Example 3.2).

1. The main result. Let $X$ be a normed space. We denote by $S_{X}$ and $B_{X}$ the unit sphere and the closed unit ball, respectively, of $X$. $L(X)$ will denote the normed algebra of all bounded linear operators on $X$, and $I_{X}$ will stand for the identity operator on $X$. Each continuous bilinear mapping from $X \times X$ into $X$ will be called a product on $X$. Each product $f$ on $X$ has a natural norm $\|f\|$ given by

$$
\|f\|:=\sup \{\|f(x, y)\|: x, y \in X,\|x\| \leq 1, \| y \leq 1\} .
$$

We denote by $\Pi(X)$ the normed space of all products on $X$. For every product $f$ on a normed space $X, f^{* * *}: X^{* *} \times X^{* *} \rightarrow X^{* *}$ will stand for the third Arens transpose of $f[\mathbf{2}]$.

Now let $u$ be a norm-one element in the normed space $X$. The set of states of $X$ relative to $u, D(X, u)$, is defined as the nonempty, convex and weak ${ }^{*}$-compact subset of $X^{*}$ given by

$$
D(X, u):=\left\{\phi \in B_{X^{*}}: \phi(u)=1\right\} .
$$

For $x$ in $X$, the numerical range of $x$ relative to $u, V(X, u, x)$ is given by

$$
V(X, u, x):=\{\phi(x): \phi \in D(X, u)\} .
$$

Thanks to the Hahn-Banach theorem, numerical ranges are contracted (respectively, preserved) under linear contractions (respectively, isometries), preserving "distinguished" elements. Indeed, if $Y$ is another 
normed space, if $v$ is a norm-one element in $Y$, and if $F$ is a linear contraction (respectively, isometry) from $X$ into $Y$ with $F(u)=v$, then for all $x$ in $X$ we have

$$
V(Y, v, F(x)) \subseteq V(X, u, x) \quad \text { respectively, } V(Y, v, F(x))=V(X, u, x) .
$$

We say that $u$ is a vertex of $B_{X}$ if the conditions $x \in X$ and $\phi(x)=0$ for all $\phi$ in $D(X, u)$ imply $x=0$. It is well known and easy to see that the vertex property for $u$ implies that $u$ is an extreme point of $B_{X}$. For $x$ in $X$, we define the numerical radius of $x$ relative to $u, v(X, u, x)$, by

$$
v(X, u, x):=\operatorname{Max}\{|\rho|: \rho \in V(X, u, x)\} .
$$

The numerical index of $X$ relative to $u, n(X, u)$, is the number given by

$$
n(X, u):=\operatorname{Max}\{r \geq 0: r\|x\| \leq v(X, u, x) \text { for all } \quad x \text { in } X\} .
$$

We note that $0 \leq n(X, u) \leq 1$ and that the condition $n(X, u)>0$ implies that $u$ is a vertex of $B_{X}$. Note also that, if $Y$ is a subspace of $X$ containing $u$, then $n(Y, u) \geq n(X, u)$. According to a result of Crabb, Duncan and McGregor [10, Theorem 3], if $A$ is a nonzero $C^{*}$ algebra with a unit $\mathbf{1}$, then $n(A, \mathbf{1})$ is equal to 1 or $1 / 2$ depending on whether or not $A$ is commutative.

Let $A$ be a complex algebra. The unital hull $A_{1}$ of $A$ is defined by $A_{1}:=A$, if $A$ has a unit, and otherwise by $A_{1}:=\mathbf{C} \mathbf{1} \oplus A$ with product

$$
(\lambda \mathbf{1}+x)(\mu \mathbf{1}+y):=\lambda \mu \mathbf{1}+(\lambda y+\mu x+x y) .
$$

In any case, $A_{1}$ is a unital complex algebra containing $A$ as an ideal. For $z$ in $A_{1}$, we denote by $T_{z}$ the linear operator on $A$ defined by $T_{z}(x):=z x$ for every $x$ in $A$. We note that, if the algebra $A$ is normed, then, for $z$ in $A_{1}$, the operator $T_{z}$ is continuous. We also note that, if $*$ is an algebrainvolution on $A$, then it extends uniquely to an algebra-involution, also denoted by $*$, on $A_{1}$, which is given by $(\lambda \mathbf{1}+x)^{*}:=\bar{\lambda} \mathbf{1}+x^{*}$. It is known that, if the algebra $A$ is a $C^{*}$-algebra, then $A_{1}$, with the involution above and the norm $\|\cdot\|$ given by $\|z\|:=\left\|T_{z}\right\|$ for $z$ in $A_{1}$ becomes a $C^{*}$-algebra containing $A$ isometrically (see for instance Lemma 12.19 in [4] and its proof). 
For every normed algebra $A, p_{A}$ will denote the natural product of $A$ and, when $A$ is endowed with an involution, $A_{s a}$ will stand for the self-adjoint part of $A$. Also, if $E$ is any set, then $B(E, A)$ will mean the normed algebra of all bounded functions from $E$ into $A$ (with point-wise operations and the supremum norm). For background on $C^{*}$-algebras, the reader is referred to $[\mathbf{1 1}]$ and $[\mathbf{2 5}]$.

Theorem 1.1. Let $A$ be a nonzero $C^{*}$-algebra. Then $n\left(\Pi(A), p_{A}\right)$ is equal to 1 or $1 / 2$ depending on whether or not $A$ is commutative.

Proof. Let $\delta$ denote either 1 or $1 / 2$ depending on whether or not $A$ is commutative. Consider the chain of linear mappings

$$
A_{1} \stackrel{F_{1}}{\longrightarrow} L(A) \stackrel{F_{2}}{\longrightarrow} \Pi(A) \stackrel{F_{3}}{\longrightarrow} \Pi\left(A^{* *}\right) \stackrel{F_{4}}{\longrightarrow} B\left(\left(A^{* *}\right)_{s a} \times\left(A^{* *}\right)_{s a}, A^{* *}\right),
$$

where $F_{1}(z):=T_{z}$ for every $z$ in $A_{1}, F_{2}(T)(x, y):=T(x y)$ for every $T$ in $L(A)$ and all $x, y$ in $A, F_{3}(f):=f^{* * *}$ for every $f$ in $\Pi(A)$, and

$$
F_{4}(g)(h, k):=e^{-i h} g\left(e^{i h}, e^{i k}\right) e^{-i k}
$$

for every $g$ in $\Pi\left(A^{* *}\right)$ and all $h, k$ in $\left(A^{* *}\right)_{s a}$. We know that $F_{1}$ and $F_{3}$ are isometries. But the same is true for $F_{2}$ (because $A$ has an approximate unit bounded by one) and also for $F_{4}$ (thanks to the Russo-Dye-Palmer theorem [4, Theorem 38.13]). Moreover, we have $F_{1}(\mathbf{1})=I_{A}, F_{2}\left(I_{A}\right)=p_{A}, F_{3}\left(p_{A}\right)=\left(p_{A}\right)^{* * *}$, which is nothing but the natural $C^{*}$-product $p_{A^{* *}}$ of $A^{* *}$, and $F_{4}\left(P_{A^{* *}}\right)=\square$, where $\square$ denotes the constant mapping equal to the unit of $A^{* *}$ on $\left(A^{* *}\right)_{s a} \times\left(A^{* *}\right)_{s a}$. Since $A_{1}$ and $B\left(\left(A^{* *}\right)_{s a} \times\left(A^{* *}\right)_{s a}, A^{* *}\right)$ are $C^{*}$-algebras with units 1 and $\square$, respectively, and they are commutative if and only if $A$ is, it follows that

$$
\begin{aligned}
\delta & =n\left(A_{1}, \mathbf{1}\right) \geq n\left(L(A), I_{A}\right) \geq n\left(\Pi(A), p_{A}\right) \\
& \geq n\left(\Pi\left(A^{* *}\right), p_{A^{* *}}\right) \geq n\left(B\left(\left(A^{* *}\right)_{s a} \times\left(A^{* *}\right)_{s a}, A^{* *}\right), \square\right)=\delta .
\end{aligned}
$$

The method of proof in the above theorem leads to other interesting consequences. As a first application the next corollary shows how, for a unital $C^{*}$-algebra $A$, numerical ranges in $\Pi(A)$ relative to $p_{A}$ can be computed in terms of numerical ranges in $A$ relative to its unit. 
Corollary 1.2. Let $A$ be a nonzero $C^{*}$-algebra with a unit 1 . Denote by $U$ the set of all unitary elements in $A$. Then, for every $f$ in $\Pi(A)$, we have

$$
\begin{aligned}
V\left(\Pi(A), p_{A}, f\right) & =\overline{\mathrm{co}}\left[\cup\left\{V\left(A, \mathbf{1}, u^{*} f(u, v) v^{*}\right): u, v \in U\right\}\right] \\
& =\overline{\mathrm{co}}\left[\cup\left\{V\left(A, \mathbf{1}, e^{-i h} f\left(e^{i h}, e^{i k}\right) e^{-i k}\right): h, k \in A_{s a}\right\}\right],
\end{aligned}
$$

where $\overline{\mathrm{co}}$ denotes closed convex hull.

Proof. The inclusion

$$
\begin{aligned}
\overline{\mathrm{co}}\left[\cup\left\{V\left(A, \mathbf{1}, e^{-i h} f\left(e^{i h}, e^{i k}\right) e^{-i k}\right): h, k \in A_{s a}\right\}\right] \\
\subseteq \overline{\mathrm{co}}\left[\cup\left\{V\left(A, \mathbf{1}, u^{*} f(u, v) v^{*}\right): u, v \in U\right\}\right]
\end{aligned}
$$

is clear, and the one

$$
\overline{\mathrm{co}}\left[\cup\left\{V\left(A, \mathbf{1}, u^{*} f(u, v) v^{*}\right): u, v \in U\right\}\right] \subseteq V\left(\Pi(A), p_{A}, f\right)
$$

follows since, for $u, v$ in $U$, the mapping $f \rightarrow u^{*} f(u, v) v^{*}$ from $\Pi(A)$ to $A$ is a linear contraction sending $p_{A}$ to 1 . Put $E:=A_{s a} \times A_{s a}$ and, for $f$ in $\Pi(A)$, let $\hat{f}$ be the element in $B(E, A)$ defined by

$$
\hat{f}(h, k):=e^{-i h} f\left(e^{i h}, e^{i k}\right) e^{-i k} .
$$

As seen in the proof of Theorem 1.1, the linear mapping $f \rightarrow \hat{f}$ from $\Pi(A)$ to $B(E, A)$ is an isometry sending $p_{A}$ to the unit $\square$ of $B(E, A)$. Therefore

$$
V\left(\Pi(A), p_{A}, f\right)=V(B(E, A), \square, \hat{f}) .
$$

But, by [22, Proposition 3], we have

$$
V(B(E, A), \square, \hat{f})=\overline{\mathrm{co}}[\cup\{V(A, \mathbf{1}, \hat{f}(h, k)):(h, k) \in E\}] .
$$

It follows that

$V\left(\Pi(A), p_{A}, f\right)=\overline{\mathrm{co}}\left[\cup\left\{V\left(A, \mathbf{1}, e^{-i h} f\left(e^{i h}, e^{i k}\right) e^{-i k}\right): h, k \in A_{s a}\right\}\right]$.

Now we handle other consequences of the method of proof of Theorem 1.1. Let $X$ be a nonzero normed space. The duality mapping of $X$ 
is the set-valued function $v \rightarrow D(X, v)$ from $S_{X}$ into $\mathcal{P}\left(X^{*}\right)$. Following [13] we say that the duality mapping of $X$ is norm-to-norm upper semi-continuous at a point $u$ of $S_{X}$ if, for every $\varepsilon>0$, there exists $\delta>0$ such that $D(X, v) \subseteq D(X, u)+\varepsilon B_{X}$ whenever $v$ is in $S_{X}$ and $\|v-u\| \leq \delta$. We note that the requirement $n(X, u)=1$ implies that the duality mapping of $X$ is upper semi-continuous at $u$ whereas, for every $0<\rho<1$, upper semi-continuity of the duality mapping of $X$ at $u$ and the requirement $n(X, u)=\rho$ are independent conditions $[\mathbf{1}, \mathrm{pp}$. 134-135].

Corollary 1.3. Let $A$ be a nonzero $C^{*}$-algebra. Then the duality mapping of $\Pi(A)$ is norm-to-norm upper semi-continuous at $p_{A}$.

Proof. With the notation in the proof of Theorem 1.1, the mapping $F_{4} \circ F_{3}$ identifies $\Pi(A)$ with a subspace of the norm-unital Banach algebra $B\left(\left(A^{* *}\right)_{s a} \times\left(A^{* *}\right)_{s a}, A^{* *}\right)$ in such a way that $p_{A}$ converts into the unit of that algebra. Then the result follows from $[\mathbf{1 9}$, Proposition 4.5] and the well-known hereditary character of the upper semi-continuity of the duality mapping.

Again let $X$ be a nonzero normed space (no distinguished normone element is chosen). The normed space numerical index, $N(X)$ of $X$ is defined by $N(X):=n\left(L(X), I_{X}\right)$. The proof of Theorem 1.1 directly gives the result of $T$. By Huruya [15], if $A$ is a nonzero $C^{*}-$ algebra, then $N(A)$ is equal to 1 or $1 / 2$ depending on whether or not $A$ is commutative. As a methodological remark, we point out that a part of our proof can be useful to clarify the first step in Huruya's original argument. In our opinion that first step (establishing the inequality $N(A) \leq 1 / 2$ for every noncommutative $C^{*}$-algebra $A$ ) is easily understood only by passing through the unit hull $A_{1}$ of $A$ in the way we have done.

We conclude this section with a variant of Huruya's theorem. For an element $x$ in an algebra $A$, we denote by $L_{x}$ the operator of left multiplication by $x$ on $A$.

Proposition 1.4. Let $A$ be a nonzero $W^{*}$-algebra, and let $A_{*}$ denote the predual of $A$. Then $N\left(A_{*}\right)$ is equal to 1 or $1 / 2$ depending on whether 
or not $A$ is commutative.

Proof. First note that, for every normed space $X$, the inequality $N\left(X^{*}\right) \leq N(X)$ holds [6, Lemma 32.7]. Assume that the $W^{*}$-algebra $A$ is commutative. Then we have $1=N(A) \leq N\left(A_{*}\right) \leq 1$. Now assume that $A$ is not commutative. Then $1 / 2=N(A) \leq N\left(A_{*}\right)$. On the other hand, by [18, Appendix 3], there exists $x$ in $S_{A}$ with $x^{2}=0$. By $[\mathbf{7}$, p. 214], for such an $x$ we have $v(A, \mathbf{1}, x)=1 / 2$ (here $\mathbf{1}$ stands for the unit of $A$ ). Since the mapping $y \rightarrow L_{y}$ from $A$ to $L(A)$ is a linear isometry sending 1 to $I_{A}$, we derive $v\left(L(A), I_{A}, L_{x}\right)=1 / 2$. By the separate weak*-continuity of the product of $A$, there exists $S$ in $L\left(A_{*}\right)$ such that $L_{x}=S^{*}$, and hence we obtain $v\left(L\left(A_{*}\right), I_{A^{*}}, S\right)=1 / 2$. It follows that $N\left(A_{*}\right)=n\left(L\left(A_{*}\right), I_{A_{*}}\right) \leq 1 / 2$.

2. Extending the results to alternative $C^{*}$-algebras. In this section we show that Theorem 1.1 remains true if the assumption of associativity for the $C^{*}$-algebra $A$ is relaxed to that of alternativity.

Alternative algebras are defined as those (not necessarily associative) algebras $A$ satisfying $x^{2} y=x(x y)$ and $y x^{2}=(y x) x$ for all $x, y$ in $A$. By Artin's theorem [26, p. 29], an algebra $A$ is alternative, (if and) only if, for all $x, y$ in $A$, the subalgebra of $A$ generated by $\{x, y\}$ is associative. As a consequence, alternative algebras are power-associative (i.e., all one-generated subalgebras are associative). Let $A$ be an alternative algebra with a unit 1 . An element $x$ in $A$ is said to be invertible if there exists $y$ in $A$ such that $x y=y x=1$. If this is the case, then the element $y$ above is uniquely determined by $x$, is called the inverse of $x$, is denoted by $x^{-1}$ and satisfies $x\left(x^{-1} z\right)=x^{-1}(x z)=z$ and $\left(z x^{-1}\right) x=\left(z x^{-1}\right) x=z$ for every $z$ in $A$ (see for instance [26, p. 38]).

A complete normed complex alternative algebra $A$ with (conjugatelinear) algebra-involution $*$ satisfying $\left\|x^{*} x\right\|=\|x\|^{2}$ for all $x$ in $A$ is called an alternative $C^{*}$-algebra. Let $A$ be an alternative $C^{*}$-algebra with a unit 1 . An element $u$ in $A$ is said to be unitary if the equalities $u u^{*}=u^{*} u=\mathbf{1}$ hold (equivalently, if $u$ is invertible in $A$ with $u^{-1}=u^{*}$ ). Such an element $u$ satisfies $\|u\|=\left\|u^{*}\right\|=1$ and $u\left(u^{*} z\right)=u^{*}(u z)=z$ and $\left(z u^{*}\right) u=(z u) u^{*}=z$ for every $z$ in $A$, so that the mappings $z \rightarrow u z$ and $z \rightarrow z u$ from $A$ to $A$ become surjective linear isometries. Distinguished unitary elements of $A$ are those of the form $e^{i x}$ where 
$x$ is in the self-adjoint part $A_{s a}$ of $A$, and it is easily shown (see for instance [8, Theorem 2.10]) that the verbatim translation of the RussoDye-Palmer theorem holds for $A$. Indeed, the equalities

$$
B_{A}=\overline{\mathrm{co}}\{u: u \text { unitary in } A\}=\overline{\mathrm{co}}\left\{e^{i h}: h \in A_{s a}\right\}
$$

are true.

Most remaining facts of the theory of (associative) $C^{*}$-algebras applied in the proof of Theorem 1.1 are also known in the wider setting of alternative $C^{*}$-algebras. For instance, if $A$ is an alternative $C^{*}$-algebra, then the bidual $A^{* *}$ of $A$, with product equal to the third Arens tranpose of the product of $A$, and involution equal to the second transpose of the involution of $A$, becomes an alternative $C^{*}$-algebra with a unit $[\mathbf{2 0}$, Corollary 1.9]. Other results needed for our purpose are directly derivable from the theory of noncommutative $J B^{*}$-algebras $[\mathbf{2 0}]$ and the fact that alternative $C^{*}$-algebras are noncommutative $J B^{*}$-algebras. Following [26, p. 141], we define noncommutative Jordan algebras as those algebras $A$ satisfying the Jordan identity $(x y) x^{2}=x\left(y x^{2}\right)$ and the flexibility condition $(x y) x=x(y x)$. Noncommutative Jordan algebras are also power associative [26, p. 141]. As a consequence of Artin's theorem, alternative algebras are noncommutative Jordan algebras. For an element $x$ in a noncommutative Jordan algebra $A$, we denote by $U_{x}$ the mapping $y \rightarrow x(x y+y x)-x^{2} y$ from $A$ to $A$. By a noncommutative $J B^{*}-$ algebra we mean a complete normed noncommutative Jordan complex algebra (say $A$ ) with algebra involution $*$ satisfying $\left\|U_{x}\left(x^{*}\right)\right\|=\|x\|^{3}$ for every $x$ in $A$. Now alternative $C^{*}$-algebras are nothing but those noncommutative $J B^{*}$-algebras which are alternative [20, Proposition 1.3]. As a first application, the extension for alternative $C^{*}$-algebras of the Crabb-Duncan-McGregor result in [10, Theorem 1] will follow from the next proposition.

Proposition 2.1 [22, Theorem 26] (see also [16, Theorem 4]). Let A be a nonzero, noncommutative $J B^{*}$-algebra with a unit $\mathbf{1}$. Then $n(A, \mathbf{1})$ is equal to 1 or $1 / 2$ depending on whether or not $A$ is associative and commutative.

Since commutative alternative complex algebras are associative $[\mathbf{2 9}$, Corollary 7.1.2], we obtain 
Corollary 2.2. Let $A$ be a nonzero alternative $C^{*}$-algebra with a unit $\mathbf{1}$. Then $n(A, \mathbf{1})$ is equal to 1 or $1 / 2$ depending on whether or not $A$ is commutative.

Now the only remaining auxiliary tools for the proof of the alternative extension of Theorem 1.1 are the following two lemmas.

Lemma 2.3. Let $A$ be a nonzero, noncommutative $J B^{*}$-algebra. Then $A_{1}$, endowed with the unique algebra involution extending the one of $A$ and the norm $\|$.$\| given by \|z\|:=\left\|T_{z}\right\|$ for all $z$ in $A_{1}$, is a noncommutative $J B^{*}$-algebra containing $A$ isometrically.

Proof. By [20, Theorem 1.7], the bidual $A^{* *}$ of $A$, with product equal to the third Arens transpose of the product of $A$, and involution equal to the second transpose of the involution of $A$, becomes a noncommutative $J B^{*}$-algebra with a unit (say $\mathbf{1}$ ). Then we can see $A_{1}$ as the normclosed $*$-invariant subalgebra of $A^{* *}$ consisting of those elements $z$ in $A^{* *}$ which can be written in the form $\lambda \mathbf{1}+x$ for some $\lambda$ in $\mathbf{C}$ and $x$ in $A$. In this way $A_{1}$ is a noncommutative $J B^{*}$-algebra containing $A$ isometrically so that, to conclude the proof, it is enough to show that the equality $\|z\|=\left\|T_{z}\right\|$ holds for every $z$ in $A_{1}$ (here $\|z\|$ means the norm of $z$ as an element of $A^{* *}$ ). Let $z$ be in $A_{1}$. Then $\left(T_{z}\right)^{* *}: A^{* *} \rightarrow A^{* *}$ and the operator of left multiplication by $z$ on $A^{* *}$, say $L_{z}^{A^{* *}}$, coincide on $A$ and are weak*-continuous (the second one, by $\left[\mathbf{2 0}\right.$, Theorem 3.5]). It follows from the weak*-density of $A$ in $A^{* *}$ that $\left(T_{z}\right)^{* *}=L_{z}^{A^{* *}}$, and hence

$$
\left\|T_{z}\right\|=\left\|\left(T_{z}\right)^{* *}\right\|=\left\|L_{z}^{A^{* *}}\right\| .
$$

Since $\|z\|=\left\|L_{z}^{A^{* *}}\right\|$, because $A^{* *}$ is a norm-unital normed algebra, we obtain $\|z\|=\left\|T_{z}\right\|$, as required.

Lemma 2.4. Let $A$ be a noncommutative $J B^{*}$-algebra and $x$ an element of $A$. Then $x$ belongs to the norm-closure of $x B_{A}$.

Proof. Take a net $\left\{y_{\lambda}\right\}$ in $B_{A}$ convergent to the unit $\mathbf{1}$ of $A^{* *}$ in the weak ${ }^{*}$ topology of $A^{* *}$. Then, by [20, Theorem 3.5], $\left\{x y_{\lambda}\right\}$ converges to $x$ in that topology. Since the net $\left\{x y_{\lambda}\right\}$ lies in $A$ and $x$ belongs to 
$A$, it follows that $\left\{x y_{\lambda}\right\}$ converges to $x$ in the weak topology of $A$, and therefore $x$ actually belongs to the weak closure of $x B_{A}$ in $A$. Finally apply that $x B_{A}$ is convex.

Now we are ready to prove the extension of Theorem 1.1 to alternative $C^{*}$-algebras.

Theorem 2.5. Let $A$ be a nonzero alternative $C^{*}$-algebra. Then $n\left(\Pi(A), p_{A}\right)$ is equal to 1 or $1 / 2$ depending on whether or not $A$ is commutative.

Proof. If $A$ is commutative, then by Theorem 1.1 we have $n\left(\Pi(A), p_{A}\right)=1$. Assume that $A$ is not commutative. We know that $A^{* *}$, with product equal to the third Arens transpose of the product of $A$, and involution equal to the second transpose of the involution of $A$, becomes an alternative $C^{*}$-algebra with a unit 1 . Moreover, since the unital hull of an alternative algebra is an alternative algebra too, it follows from Lemma 2.3 that $A_{1}$ is an alternative $C^{*}$-algebra in such a way that the mapping $z \rightarrow T_{z}$ from $A_{1}$ to $L(A)$ is an isometry. Now, consider the chain of linear mappings

$$
A_{1} \stackrel{F_{1}}{\longrightarrow} L(A) \stackrel{F_{2}}{\longrightarrow} \Pi(A) \stackrel{F_{3}}{\longrightarrow} \Pi\left(A^{* *}\right) \stackrel{F_{4}}{\longrightarrow} B\left(\left(A^{* *}\right)_{s a} \times\left(A^{* *}\right)_{s a}, A^{* *}\right),
$$

where $F_{1}, F_{2}$ and $F_{3}$ are defined verbatim as in the proof of Theorem 1.1 , whereas $F_{4}$ is determined by the equality

$$
F_{4}(g)(h, k):=e^{-i h}\left(g\left(e^{i h}, e^{i k}\right) e^{-i k}\right)
$$

for every $g$ in $\Pi\left(A^{* *}\right)$ and all $h, k$ in $\left(A^{* *}\right)_{s a}$. At this time, the isometric character of $F_{1}$ and $F_{3}$ is not in doubt. But $F_{2}$ and $F_{4}$ are also isometries. Indeed, apply Lemma 2.4 for the case of $F_{2}$ and, concerning $F_{4}$, keep in mind the extended Russo-Dye-Palmer theorem together with the fact that left and right multiplications by unitary elements on $A^{* *}$ are isometries. On the other hand, the equalities $F_{1}(\mathbf{1})=I_{A}$, $F_{2}\left(I_{A}\right)=p_{A}, F_{3}\left(p_{A}\right)=p_{A^{* *}}$ are clear, whereas $F_{4}\left(p_{A^{* *}}\right)=\square$ follows from Artin's theorem. Since the unital alternative $C^{*}$-algebras $A_{1}$ and $B\left(\left(A^{* *}\right)_{s a} \times\left(A^{* *}\right)_{s a}, A^{* *}\right)$ are not commutative, it follows from 
Corollary 2.2 that

$$
\begin{aligned}
\frac{1}{2} & =n\left(A_{1}, \mathbf{1}\right) \geq n\left(L(A), I_{A}\right) \geq n\left(\Pi(A), p_{A}\right) \\
& \geq n\left(\Pi\left(A^{* *}\right), p_{A^{* *}}\right) \geq n\left(B\left(\left(A^{* *}\right)_{s a} \times\left(A^{* *}\right)_{s a}, A^{* *}\right), \square\right)=\frac{1}{2} .
\end{aligned}
$$

It is worth mentioning that Corollary 1.3 remains true, without any change in its formulation and proof, in the wider setting of alternative $C^{*}$-algebras. Concerning Corollary 1.2, it needs a light retouching of formulation in order to remain valid in our new context. Namely, if $A$ is a nonzero alternative $C^{*}$-algebra with a unit $\mathbf{1}$, and if $U$ denotes the set of all unitary elements in $A$, then, for every $f$ in $\Pi(A)$, we have

$$
\begin{aligned}
V\left(\Pi(A), p_{A}, f\right) & =\overline{\mathrm{co}}\left[\cup\left\{V\left(A, \mathbf{1}, u^{*}\left(f(u, v) v^{*}\right)\right): u, v \in U\right\}\right] \\
& =\overline{\mathrm{co}}\left[\cup\left\{V\left(A, \mathbf{1}, e^{-i h}\left(f\left(e^{i h}, e^{i k}\right) e^{-i k}\right)\right): h, k \in A_{s a}\right\}\right] .
\end{aligned}
$$

Let us also note that the proof of Theorem 2.5 contains an extended Huruya theorem. Indeed, if $A$ is an alternative $C^{*}$-algebra, then $N(A)$ is equal to 1 or $1 / 2$ depending on whether or not $A$ is commutative. In fact, the next proposition provides us with a more general result. For a complex normed space $X$ and a norm-one element $u$ in $X$, we write

$$
H(X, u):=\{x \in X: V(X, u, x) \subseteq \mathbf{R}\} .
$$

Proposition 2.6. Let $A$ be a nonzero noncommutative $J B^{*}$-algebra. Then $N(A)$ is equal to 1 or $1 / 2$ depending on whether or not $A$ is associative and commutative.

Proof. Assume that $A$ is associative and commutative. Then $A$ is a commutative $C^{*}$-algebra and, therefore, we have $N(A)=1$. Now assume that $A$ fails to be associative or commutative. By the already applied Theorem 1.7 in $[\mathbf{2 0}], A^{* *}$ is a unital noncommutative $J B^{*}$-algebra in a natural way. Moreover, by Lemma $2.3, A_{1}$ is a noncommutative $J B^{*}$-algebra in such a way that the mapping $z \rightarrow T_{z}$ from $A_{1}$ to $L(A)$ becomes an isometry. Consider the chain of linear mappings

$$
A_{1} \stackrel{G_{1}}{\longrightarrow} L(A) \stackrel{G_{2}}{\longrightarrow} L\left(A^{* *}\right) \stackrel{G_{3}}{\longrightarrow} B\left(\left(A^{* *}\right)_{s a}, A^{* *}\right),
$$


where $G_{1}(z):=T_{z}$ for every $z$ in $A_{1}, G_{2}(T):=T^{* *}$ for every $T$ in $L(A)$, and

$$
G_{3}(S)(h):=e^{-i L_{h}} S\left(e^{i h}\right)
$$

for every $S$ in $L\left(A^{* *}\right)$ and all $h$ in $\left(A^{* *}\right)_{s a}$. We know that $G_{1}$ and $G_{2}$ are isometries. To see that the same is true for $G_{3}$, apply the Russo-DyePalmer-type theorem for noncommutative $J B^{*}$-algebras $[\mathbf{2 7}$, Corollary 2.4], and note that, if $h$ is in $\left(A^{* *}\right)_{s a}$, then $h$ belongs to $H\left(A^{* *}, \mathbf{1}\right)[\mathbf{2 8}$, Theorem $7(\mathrm{a})]$, so $L_{h}$ belongs to $H\left(L\left(A^{* *}\right), I_{-A^{* *}}\right)$ and so $e^{-i L_{h}}$ is an isometry [5, Lemma 5.2]. Since we have $G_{1}(\mathbf{1})=I_{A}, G_{2}\left(I_{A}\right)=I_{A^{* * *}}$, and $G_{3}\left(I_{A^{* *}}\right)=\square$ (where $\square$ denotes the constant mapping equal to the unit of $A^{* *}$ on $\left.\left(A^{* *}\right)_{s a}\right)$, it follows from Proposition 2.1 that

$$
\begin{aligned}
\frac{1}{2} & =n\left(A_{1}, \mathbf{1}\right) \geq n\left(L(A), I_{A}\right) \geq n\left(L\left(A^{* *}\right), I_{A^{* *}}\right) \\
& \geq n\left(B\left(\left(A^{* *}\right)_{s a}, A^{* *}\right), \square\right)=\frac{1}{2} .
\end{aligned}
$$

Remark 2.7. (i) Proposition 2.6 was formulated in [16, Theorem 5] as a direct consequence of [20, Theorem 1.7], the particular case of that proposition for unital algebras [22, Corollary 33], and the claim in [12] that, for every normed space $X$, the equality $N\left(X^{*}\right)=N(X)$ holds. As a matter of fact, the proof of the claim in $[\mathbf{1 2}]$ never appeared, and the question if for an arbitrary normed space $X$ the equality $N\left(X^{*}\right)=N(X)$ holds remains an open problem among people interested in the field.

(ii) Let $A$ be a noncommutative $J B^{*}$-algebra. Since $A^{* *}$ is a noncommutative $J B^{*}$-algebra and $A^{* *}$ is associative and commutative if (and only if) the same is true for $A$ [20, Theorem 1.7], it follows from Proposition 2.6 that the equality $N\left(X^{*}\right)=N(X)$ holds for $X$ equal to either $A$ or $A^{*}$.

Noncommutative $J B W^{*}$-algebras are defined as those noncommutative $J B^{*}$-algebras which are dual Banach spaces. If $A$ is a noncommutative $J B W^{*}$-algebra, then $A$ has a unit, the predual of $A$ is unique, and the product of $A$ is separately weak*-continuous $[\mathbf{2 0}, \mathrm{p}$. 104]. Our concluding result in this section shows that the equality $N\left(X^{*}\right)=N(X)$ also holds for $X$ equal to the predual $A_{*}$ of every noncommutative $J B W^{*}$-algebra $A$. Replacing Huruya's classical theorem, 
[18, Appendix 3, Theorem B] and [7, Corollary 2, p. 214] by Proposition 2.6, [16, Theorem 1] and [22, Proposition 29], respectively, the proof is almost the same as that of Proposition 1.4, and therefore it is omitted.

Proposition 2.8. Let $A$ be a nonzero noncommutative $J B W^{*}$ algebra. Then $N\left(A_{*}\right)$ is equal to 1 or $1 / 2$ depending on whether or not $A$ is associative and commutative.

3. Discussing the results. Keeping in mind that most auxiliary results applied to prove Theorem 2.5 are also valid in the setting of noncommutative $J B^{*}$-algebras, one can suspect in a first instance that, if $A$ is a nonzero noncommutative $J B^{*}$-algebra, then $n\left(\Pi(A), p_{A}\right)$ is equal to 1 or $1 / 2$ depending on whether or not $A$ is associative and commutative. As a matter of fact, this suspicion is very far from being right. Indeed, it is very easy to provide us with noncommutative $J B^{*}$ algebras $A$ whose products $p_{A}$ are not extreme points of $B_{\Pi(A)}$, so that $p_{A}$ cannot be a vertex of $B_{\Pi(A)}$, and hence $n\left(\Pi(A), p_{A}\right)=0$. For instance, if $B$ is a $C^{*}$-algebra which fails to be commutative, if $\lambda$ is a real number with $0<\lambda<1$, and if we replace the product $x y$ of $B$ by the one

$$
(x, y) \longrightarrow \lambda x y+(1-\lambda) y x,
$$

then we obtain a noncommutative $J B^{*}$-algebra (say $A$ ) whose product is not an extreme point of $B_{\Pi(A)}$. With $\lambda=1 / 2$ in the above construction we even obtain a (commutative) $J B^{*}$-algebra with such a pathology. Since, on the other hand, we do not know noncommutative $J B^{*}$-algebras $A$ whose products have the vertex property in $\Pi(A)$ other than alternative $C^{*}$-algebras, we dare to formulate the following conjecture.

Conjecture 3.1. A noncommutative $J B^{*}$-algebra $A$ is alternative if (and only if) the product of $A$ is a vertex of the closed unit ball of $\Pi(A)$.

Concerning the above conjecture, the only remarkable fact that we know up to date is that, if we relax the vertex property to the extreme point property, then the answer is negative. This is shown by the next 
example. Following $[\mathbf{2 6}$, p. 50], we say that a complex algebra $A$ is quadratic if $A$ has a unit $\mathbf{1}, A \neq \mathbf{C 1}$ and $x^{2}$ belongs to the linear hull of $\{\mathbf{1}, x\}$ for all $x$ in $A$. A Vidav (in short, $V$-) algebra is a norm-unital complete normed complex algebra $A$ satisfying $A=H(A, \mathbf{1}) \oplus i H(A, 1)$ where 1 stands for the unit of $A$. For such an algebra $A$, the so-called natural involution of $A$, given by $(x+i y)^{*}:=x-i y, x, y \in H(A, \mathbf{1})$, is an algebra-involution $[\mathbf{2 3}$, Theorem 1].

Example 3.2. Let $A$ be the (commutative) $J B^{*}$-algebra whose Banach space is the $*$-invariant subspace of the $C^{*}$-algebra $M_{2}(\mathbf{C})$ given by

$$
\left\{\left(\begin{array}{ll}
\alpha & \beta \\
\gamma & \alpha
\end{array}\right): \alpha, \beta, \gamma \in \mathbf{C}\right\}
$$

and whose product is the one $\circ$ defined by

$$
x \circ y:=\frac{1}{2}(x y+y x)
$$

for all $x, y$ in $A$. Since $A$ is commutative and fails to be associative, it follows that $A$ is not alternative. We will prove that $p_{A}$ is an extreme point of $B_{\Pi(A)}$. To this end we take $f, g$ in $B_{\Pi(A)}$ and $0<\lambda<1$ such that $\lambda f+(1-\lambda) g=p_{A}$, and we proceed to show that $f=p_{A}$, say. If 1 denotes the unit of $A$ then, by [20, Lemma 1.5], we have $f(x, \mathbf{1})=f(\mathbf{1}, x)=x$ for every $x$ in $A$. In this way $\mathbf{1}$ is a unit for the complete normed complex algebras (say $B$ and $B^{+}$) consisting of the Banach space of $A$ and the products $f$ and

$$
f^{+}:(x, y) \longrightarrow \frac{1}{2}(f(x, y)+f(y, x))
$$

respectively. Since $A$ is a $V$-algebra and Vidav's requirement involves only the Banach space and the unit, $B$ and $B^{+}$are also $V$-algebras whose natural (automatically algebra-) involutions coincide with the $J B^{*}$-involution of $A$. Since $A$ and $B^{+}$are commutative $V$-algebras, and the mapping $F: x \rightarrow x$ from $A$ to $B^{+}$is a surjective linear isometry preserving the units, the argument in the proof of the implication (i) $\Rightarrow$ (ii) in $[\mathbf{1 7}$, Lemma 6$]$ shows that $F$ is an algebra isomorphism, so $f^{+}$ coincides with $p_{A}$, and hence $B^{+}=A$. Now, by [17, Theorem 8] and [20, Proposition 1.2], $B$ is a noncommutative $J B^{*}$-algebra and, since $A$ is quadratic and squares in $B$ and $B^{+}$coincide, $B$ is quadratic too. 
Then, as a consequence of [21, Theorem 3.2], there exist a real Hilbert space $(E,(. \mid)$.$) and an anti-commutative product \wedge$ on $E$ satisfying $(x \mid x \wedge y)=0$ for all $x, y$ in $E$ such that, if we consider the real algebra $C$ consisting of the vector space $\mathbf{R} \mathbf{1} \oplus E$ and the product

$$
(\lambda \mathbf{1}+x)(\mu \mathbf{1}+y):=(\lambda \mu-(x \mid y)) \mathbf{1}+(\lambda y+\mu x+x \wedge y)
$$

then, as a complex algebra, $B$ is nothing but the complexification of $C$. Since $A$ is three-dimensional over $\mathbf{C}, E$ must be two-dimensional over R. Let $\{u, v\}$ be a basis of $E$. Then we have $(u \mid u \wedge v)=(v \mid u \wedge v)=0$, so $u \wedge v=0$ and hence $\wedge$ is identically zero on $E$. Therefore, $B$ is commutative, so that $B=B^{+}$. Since we know that $B^{+}=A$, we obtain $B=A$. This means $f=p_{A}$ as required.

Acknowledgments. Part of this work was done while the third author was visiting the University of Almería. He is grateful to the Department of Algebra and Mathematical Analysis of that university for its hospitality and support. The authors also thank M.V. Velasco for several valuable remarks.

\section{REFERENCES}

1. C. Aparicio, F. Ocaña, R. Paya and A. Rodriguez, A non-smooth extension of Fréchet differentiability of the norm with applications to numerical ranges, Glasgow Math. J. 28 (1986), 121-137.

2. R. Arens, The adjoint of a bilinear operation, Proc. Amer. Math. Soc. 2 (1951), 839-848.

3. H.F. Bohnenblust and S. Karlin, Geometrical properties of the unit sphere of a Banach algebra, Ann. Math. 62 (1955), 217-229.

4. F.F. Bonsall and J. Duncan, Complete normed algebras, Springer-Verlag, Berlin, 1973.

5. - Numerical ranges of operators on normed spaces and of elements of normed algebras, London Math. Soc. Lecture Note Ser. 2, Cambridge, 1971.

6. — Numerical ranges II, London Math. Soc. Lecture Note Ser. 10, Cambridge, 1973.

7. R. Bouldin, The numerical range of a product, II, J. Math. Anal. Appl. 33 (1971), 212-219.

8. R.B. Braun, A Gelfand-Neumark theorem for $C^{*}$-alternative algebras, Math. Z. 185 (1984), 225-242.

9. M. Cabrera and A. Rodriguez, New associative and nonassociative GelfandNaimark theorems, Manuscripta Math. 79 (1993), 197-208. 
10. M.J. Crabb, J. Duncan and C.M. McGregor, Characterizations of commutativity for $C^{*}$-algebras, Glasgow Math. J. 15 (1974), 172-175.

11. J. Dixmier, Les $C^{*}$-algèbres et leurs représentations, Gautiers-Villars, Paris, 1969.

12. J. Duncan, Review of [19], Math. Rev. 87e: 46067.

13. J.R. Giles, D.A. Gregory and B. Sims, Geometrical implications of uppersemicontinuity of the duality mapping on a Banach space, Pacific J. Math. 79 (1978), 99-108.

14. G. Horn, Coordinatization theorem for $J B W^{*}$-triples, Quart. J. Math. Oxford 38 (1987), 321-335.

15. T. Huruya, The normed space numerical index of $C^{*}$-algebras, Proc. Amer. Math. Soc. 63 (1977), 289-290.

16. B. Iochum, G. Loupias and A. Rodriguez, Commutativity of $C^{*}$-algebras and associativity of $J B^{*}$-algebras, Math. Proc. Cambridge Philos. Soc. 106 (1989), 281-291.

17. A.M. Kaidi, J. Martinez and A. Rodriguez, On a non-associative VidavPalmer theorem, Quart. J. Math. Oxford 32 (1981), 435-442.

18. I. Kaplansky, Rings of operators, Benjamin, New York, 1968.

19. J. Martinez, J.F. Mena, R. Paya and A. Rodriguez, An approach to numerical ranges without Banach algebra theory, Illinois J. Math. 29 (1985), 609-625.

20. R. Paya, J. Perez and A. Rodriguez, Non-commutative Jordan $C^{*}$-algebras, Manuscripta Math. 37 (1982), 87-120.

21. - Type I factor representations of non-commutative JB*-algebras, Proc. London Math. Soc. 48 (1984), 428-444.

22. A. Rodriguez, A Vidav-Palmer theorem for Jordan $C^{*}$-algebras and related topics, J. London Math. Soc. 22 (1980), 318-332.

23. - Non-associative normed algebras spanned by Hermitian elements, Proc. London Math. Soc. 47 (1983), 258-274.

24. Isometries and Jordan-isomorphisms onto $C^{*}$-algebras, J. Operator Theory 40 (1998), 71-85.

25. S. Sakai, $C^{*}$ - and $W^{*}$-algebras, Springer-Verlag, Berlin, 1971.

26. R.D. Schafer, An introduction to nonassociative algebras, Academic Press, New York, 1966.

27. J.D.M. Wright and M.A. Youngson, A Russo Dye theorem for Jordan $C^{*}$ algebras, in Functional analysis: Surveys and recent results (K.D. Bierstedt and F. Fuchssteiner, eds.), North-Holland Math. Stud. 27, Amsterdam, 1977.

28. M.A. Youngson, A Vidav theorem for Banach Jordan algebras, Math. Proc. Cambridge Philos. Soc. 84 (1978), 263-272.

29. K.A. Zhevlakov, A.M. Slin'ko, I.P. Shestakov and A.I. Shirshov, Rings that are nearly associative, Academic Press, New York, 1982. 
Departamento de Algebra y Análisis Matemático, Universidad de Almería, Facultad de Ciencias Experimentales, 04120-Almería, Spain

E-mail address: elamin@ual.es

Departamento de Algebra y Análisis Matemático, Universidad de Almería, Facultad de Ciencias Experimentales, 04120-Almería, Spain

E-mail address: amorales@ual.es

Departamento de Análisis Matemático, Universidad de Granada, Facultad de Ciencias, 18071-Granada, Spain

E-mail address: apalacio@ugr.es 